\title{
Of mice and men: Virtual Hebb-Williams mazes permit comparison of spatial learning across species
}

\author{
DAVID I. SHORE \\ McMaster University, Hamilton, Ontario, Canada \\ and Dalhousie University, Halifax, Nova Scotia, Canada \\ LIANNE STANFORD \\ Dalhousie University, Halifax, Nova Scotia, Canada \\ W. JOSEPH MACINNES \\ DalTech, Halifax, Nova Scotia, Canada \\ and \\ RAYMOND M. KLEIN and RICHARD E. BROWN \\ Dalhousie University, Halifax, Nova Scotia, Canada
}

\begin{abstract}
We developed a computer-generated virtual environment to test humans, for the first time, on the Hebb-Williams mazes. The goal was to provide a standardized test that could be used to directly compare human performance with that of C57BL/6J mice performing in real versions of the mazes. Such a comparison seems crucial if conclusions regarding genetic manipulations of rodents are to be mapped onto human cognitive disorders. The learning curves across species were strikingly similar, lending support to the rodent model of human spatial memory. Humans learned faster than rodents in both the acquisition and the test portions of the protocol, and females of both species were less efficient in solving these problems than males. These results represent the first modern comparison of human and rodent learning that uses the same test of spatial problem solving.
\end{abstract}

Maze learning has been used as a measure of spatial intelligence for approximately 100 years (Clements \& Brown, 1999; Olton, 1979; Olton \& Papas, 1979). The Hebb-Williams mazes (Hebb \& Williams, 1946; Rabinovitch \& Rosvold, 1951) consist of a standardized set of problems that have been used to test a wide range of species, including rats (seven strains), cats, rabbits, ferrets, possum, mice (at least a dozen strains), voles, sheep, rams, lambs, goldfish, leghorn chicks, bears, raccoons, baby monkeys, and even cows. But not, until now, humans. Primarily to remedy this omission, and to permit the comparison of human and animal performance on this widely used and well-understood set of problems, we have de-

This research was supported by a Killam Post-Doctoral Fellowship and a Rotman Research Fellowship to D.I.S., by a Dalhousie University Graduate Fellowship and a Canadian Institutes of Health Research/ K.M. Hunter Doctoral Research Award to L.S., and by NSERC operating grants to R.M.K. and R.E.B. The original idea for this paper was developed after the 1997 Psychology In-House Convention at Dalhousie University. We also thank Patti Guidry for assistance in collecting the human data and Patti Guidry, Jennifer Stapleton, Kelly Throp, Allison Clarke, Mark Marshall, John Vessey, Brett Passi, Jeff Collins, and Rosanna Rhaman for assistance in collecting mouse data. Also, thanks to Trudy A. Shore for help with computer graphics and WWW components. Correspondence concerning this article should be addressed to D. I. Shore, Department of Psychology, McMaster University, 1280 Main St. West, Hamilton, ON, L8S 4K1 Canada (www.mcmaster.ca / dshore; e-mail: dshore@mcmaster.ca). veloped a computer-generated virtual version of the HebbWilliams maze problems.

Recent studies using virtual environments (Darken \& Sibert, 1996) have begun to address several issues involved in route learning, cognitive map making, and efficient navigation. Because many of these projects use novel paradigms and environments (e.g., Gron, Wunderlich, Spitzer, Tomczak, \& Riepe, 2000; Moffat, Hampson, \& Hatzipantelis, 1998), there is nothing with which to compare the newly generated human performance data. Moreover, researchers often cannot replicate or extend findings because of the unavailability of specialized equipment (Gillner \& Mallot, 1998) or the lack of published maze details (Gron et al., 2000). In contrast, the layout and protocol we use have been standardized (Rabinovitch \& Rosvold, 1951), and cross-species comparisons are facilitated by the large literature described above. Several recent studies using a virtual Morris water maze (Astur, Ortiz, \& Sutherland, 1998; Jacobs, Laurance, \& Thomas, 1997; Jacobs, Thomas, Laurance, \& Nadel, 1998; Sandstrom, Kaufman, \& Huettel, 1998) also take advantage of an existing literature to facilitate cross-species comparisons but are limited because this task consists of only one, unidimensional problem. The Hebb-Williams mazes vary in difficulty and, therefore, provide a richer pattern of results.

The need to evaluate mouse models of human cognitive disorders provided further impetus for this research. Recent advances in genetic manipulation involving knock- 
out, transgenic, and mutant mice have enabled the development of mouse models of human diseases, such as Alzheimer's, Parkinson's, and Huntington's. Much research has focused on comparing the neuropathology of mouse models of these diseases with that of human patients. Little is known, however, about the behavioral deficits in these mice (Emilien, Maloteaux, Beyreuther, \& Masters, 2000; van Leuven, 2000). In light of the advances on the genetic front, the development of suitable behavioral tests for genetically modified mice is a priority. Such tests of mouse behavior must target cognitive functions that appear deranged in the human form of a disease. It would be ideal, for example, if disordered mice and human patients, and their corresponding controls, could be tested on species-appropriate tests that are otherwise matched and standardized. As a test of spatial learning and performance, the Hebb-Williams mazes would be ideally suited for this purpose-if humans could be tested on them.

\section{METHOD}

Mice were run using a standard Hebb-Williams protocol modified for the species (Meunier, Saint-Marc, \& Destrade, 1986; Rabinovitch \& Rosvold, 1951). There were 10 male and 10 female mice. The mice were food restricted to approximately $85 \%$ of their ad-lib body weight as determined on the 5 days prior to running. They were habituated to the maze environment devoid of any internal walls for one 20-min session per day for 5 days. During the last two sessions, the food cup was baited with Froot Loops cereal pieces (Kellogg's Canada, Etobicoke, ON), and the mice had ad-lib access to this for the entire 20-min session. After the habituation sessions, the mice were trained in the acquisition mazes two sessions per day. The six acquisition mazes were presented in the same order, with one trial per maze in one of two session styles (either A-B-C-D-E-F-A-B-C or D-E-F-A-B-C-D-E-F) so that each maze was run three times per day. When the mice could complete two consecutive sessions in less than $60 \mathrm{sec}$ each, they advanced to the test mazes. During each of the two daily sessions, one test maze was run for five trials. Both the amount of time for the mouse to run through the maze from start to goal box and the number of errors for each trial were recorded. The maze patterns are highlighted on the top of Figure 1. The dark bold lines outline the maze walls, and the dotted lines indicate invisible error lines. An error was scored (Rabinovitch \& Rosvold, 1951) when the front two paws of the mouse entered into one of these zones.

The human participants were 20 (10 male) Dalhousie University undergraduates who performed all acquisition blocks and test mazes in one sitting that took approximately $2 \mathrm{~h}$. The mazes were presented in the same order as that for the mice, with the exception that a blank maze (devoid of internal walls) was inserted after every five trials to inform the subject that a new maze was about to begin. All the experiments were conducted on a Pentium based computer system (without three-dimensional [3-D] hardware acceleration) with a 17-in. Viewsonic monitor. Mazes were rendered using OpenGL in full-screen mode at a resolution of $640 \times 480$. In order to create a pseudo real-time environment, the Windows messaging loop was removed to eliminate unwanted interruptions from the operating system, and the keyboard was monitored directly by the program to improve response time and allow for simultaneous keypresses.

As seen in Figure 2, each maze consisted of a $6 \times 6$ room, with $1 \times 1$ alcoves at the corners of the origin (start) and the destination (goal). Walls were rendered using thin, textured rectangles, and floors using red and black square tiles. The roof of the goal alcove was painted red, and the start alcove roof was green. Controls for the maze were the standard keyboard arrow keys that maintained a constant velocity of $12 \mathrm{~km} / \mathrm{h}$ (forward, backward) and a turn rate of $50^{\circ}$ per second (left, right). Based on a viewing height of $5 \mathrm{ft} 6$ in., the projection of the entire maze appeared to measure $20 \mathrm{~m}^{2}$, and the diagonal straight line from start to finish was perceived as 28.3 $\mathrm{m}$. The length of time from start to goal, errors, and paths taken were recorded for each trial of each maze. Errors for human subjects were scored when the virtual person-essentially coded as a single point in space-crossed an error line. Note that the walls for the mice were made of black Plexiglas, whereas the walls for the human subjects were textured to provide a better sense of depth. Two digital images of the inside of the virtual maze can be seen on line (www. momaster.ca/dshore/hebb).

\section{RESULTS}

In the first stage of our testing, both human and rodent subjects completed acquisition training until a fixed criterion (158 sec for humans and $60 \mathrm{sec}$ for mice) was met on two consecutive sessions. Time spent in the acquisition environment (across sessions) was less for males than for females $(1,746$ vs. $2,526 \mathrm{sec} ; U=156, p<.05)$, regardless of species, and was more for humans than for mice, regardless of gender (3,426 vs. $686 \mathrm{sec} ; U=12, p<.0001)$. There was no gender $\times$ species interaction $(F<1.0)$.

Scores from the 12 test mazes were standardized to permit comparisons between species, since humans took longer to complete any given maze than the mice but made only one third as many errors (Figure 3 ). In addition, rather than emphasizing either time or errors (Davenport, Hagquist, \& Rankin, 1970; Pollard \& Sampson, 1961), we combined them into one performance measure that was standardized for each species separately (see Figure 3). In itself, this is an important innovation, since most previous work on the maze has reported only errors that were arbitrarily defined (see Rabinovitch \& Rosvold, 1951, for rules) and have little ecological validity. The measure described in Figure 3 could be applied to the previous literature and could be used to compare findings from different labs. The data in Figure 3 (panel C) indicate that humans learn at a faster rate than mice $[F(4,160)=2.77$, $p<.05]$, reaching average performance by the second trial, whereas the mice required an additional trial to better the average performance. There was also a robust main effect of gender $[F(1,40)=22.06, p<.005]$, which did not interact with species $[F(1,40)=1.75$, n.s. $]$. This indicates that females were, overall, less efficient at solving these spatial problems than their male counterparts. This difference is most striking on the first trial $[F(1,40)=$ $6.24, p<.025]$. For humans, this difference is predominantly seen in the amount of time spent pausing (compare the upper portion of the bars in the left panel of Figure $3 \mathrm{~A}$ ). An examination across the mazes (Figures 1C and 1D) confirms that females, of both species, were less efficient on the first trial -9 out of 12 mazes for mice and 10 out of 12 mazes for humans.

The analysis across the 12 mazes (Figure 1) assesses the ability to generalize from one species to another. 


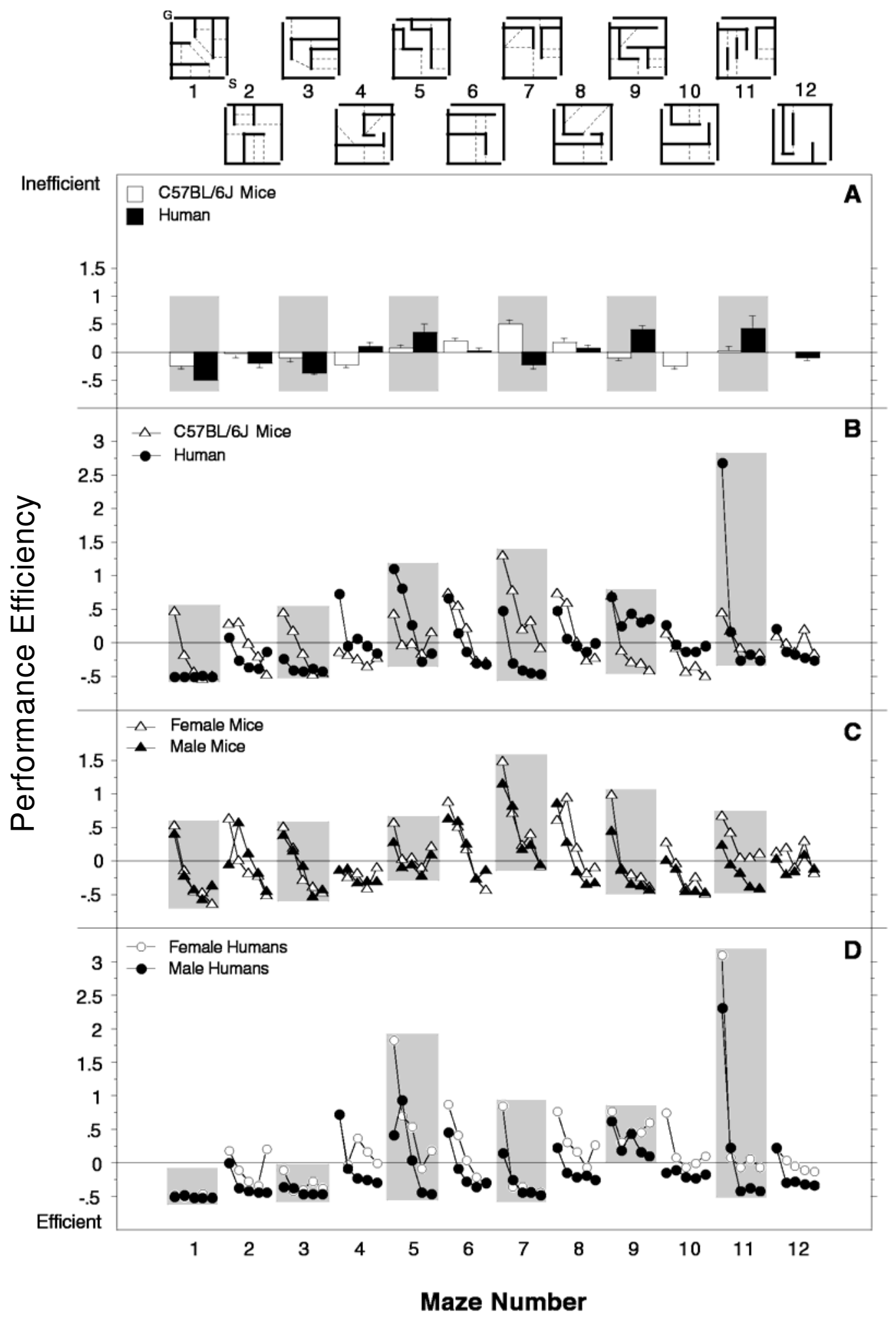

Figure 1. Performance efficiency across the 12 mazes (illustrated at the top of the figure). Panel A shows average performance collapsed across the five trials. Relatively speaking, mice outperformed humans on some mazes $(4,5$, and 9$)$, whereas the reverse was true on other mazes $(1,3$, and 7$)$. Panel $B$ compares performance across trials for the two species and further illustrates that the overall pattern of data across trials is very similar for both species. The data for mice (C) and humans (D) are presented separately for each gender. 



4

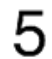

\section{Trial}

Figure 2. Path analysis for human females (red traces) and human males (blue traces) running in Maze 5 (A) and Maze 11 (B). The paths of all the subjects are superimposed to yield a qualitative assessment of learning across trials. The black dots represent locations where subjects paused for any duration. Apparent in this figure is the robust gender difference- the women were overall less efficient in traversing the mazes than were the men $[F(1,19)=21.5$, $p<.025]$. In addition, the women paused more than did the men $[F(1,19)=14.6, p<.0025]$. This difference is most apparent in the first trial of both mazes shown here. This difference is maintained to Trials 4 and 5 of Maze 5 , where several women had difficulty with the two blind alleys. In Trial 5 of Maze 11, there are no qualitative differences between the men and the women. It is interesting to note that one member of each gender maintained a path that entered a blind alley. Performance of 6 subjects (the best, median, and worst male and female) running in Maze 5 can be seen unfolding on the World-Wide Web (www.mcmaster.ca/dshore/hebb). This qualitative data further emphasize the gender differences observed above.

There was a robust interaction between species and maze $[F(11,440)=4.55, p<.0001]$, which would seem to undermine the claim of similar performance on these mazes by the two species (see Figure A). However, closer inspection of the learning performance across trial within each maze indicates clear similarities. In particular Mazes
6,8 , and 12 show almost identical levels of learning and similar levels of asymptotic performance. Mazes 7, 9, and 10 reveal a similarity in learning, with an overall different level of asymptotic performance. Mazes 1, 3, 4, and 11 show a pattern in which asymptotic performance is identical but there is a clear learning difference. The re- 


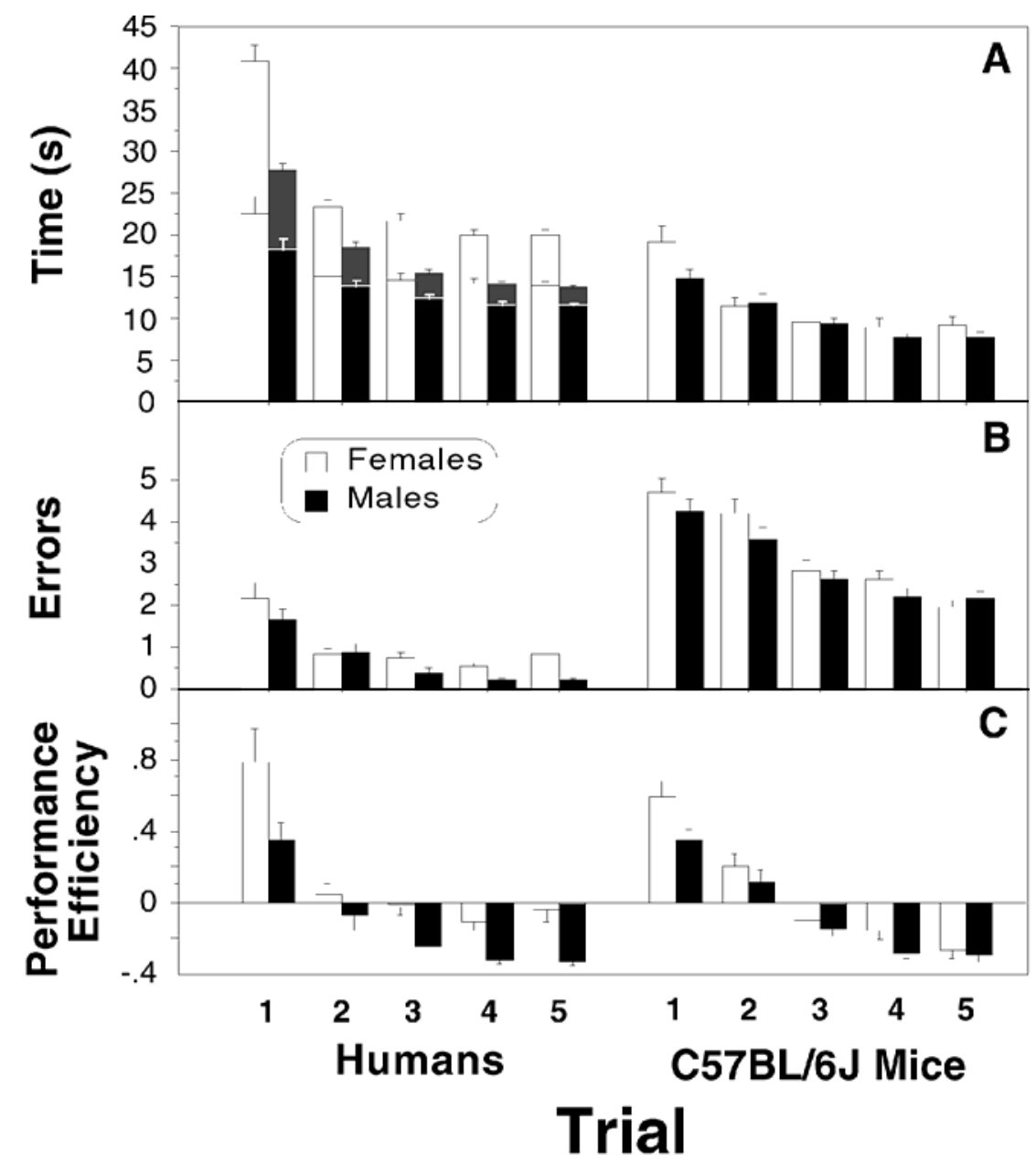

Figure 3. Performance of humans and C57B L/6J mice on each of five trials averaged over the 12 mazes. Maze completion time $(A)$ and total number of errors $(B)$ are combined into a standardized performance efficiency measure $(C)$ to allow comparisons across species. Humans were slower overall $[F(1,40)=84.5, p<.0001]$ but made fewer errors $[F(1,40)=179.0, p<.0001]$ than the mice. Each subject's time and error score were converted to $Z$ scores using the overall grand means and standard deviations from all subjects from the same species. Averaging the two $Z$ scores (whereby increases in time and errors are weighted equally) provides a composite measure wherein large numbers reflect relatively poor performance. Human time scores (panel $\mathrm{A}$, left) are divided into two components-time spent in motion (bottom portion of each bar) and "inspection time," or time spent pausing (top portion of each bar) - which together represent the total time. Because the sessions with mice were not videotaped, we are unable to make this division with the time scores for the mice. Error bars represent the standard error of the mean between subjects.

maining mazes ( 2 and 5 ) show differences in both learning and performance. Thus, despite the robust interaction across maze, many similarities were also observed.

\section{DISCUSSION}

There are three main findings from this study. Humans learn faster than mice, there are systematic similarities and differences in maze-running performance between the species, and, males are more efficient at solving these spatial problems than females. ${ }^{1}$ These data make clear contributions to three areas of research-cross-species comparison of spatial maze learning, the utility of virtual environments for testing human subjects, and gender differences in way finding.

There are clear similarities in learning on a number of mazes; however, there are also some differences. In order to fully understand the nature of this interaction, species differences must be examined in relation to the particular maze layout. Consider Maze 1 (See Figure 1), for which 
humans are at asymptotic performance from the first trial and mice require three trials for performance to stabilize. Examination of this layout reveals that the goal box is visible from the start box and the humans take advantage of this by moving straight through the maze. The mice on the other hand may not rely on visual information to the same extent as humans and thus may not avail themselves of this cue. In addition, mice tend to avoid open spaces (i.e., the middle of the maze), whereas humans do not have this aversion and thus are more likely to move directly to the seen goal box. Although similar reasoning could be applied to the other mazes, we wish to focus instead on the mazes for which species differences in performance were minimal (e.g., Mazes 6, 7, 8, 9,10 , and 12) and propose that this subset of the HebbWilliams mazes may represent a rodent spatial-learning test that can be directly linked to human performance. It is important to note that the standardization procedure used throughout the history of the maze has included all of the mazes, so any use of only a subset should be done with caution.

Future research should focus on this subset of mazes with both patient populations and different strains of mutant mice, in order to match both brain morphology and behavioral anomalies. It is important to consider whether different strategies or motivations are involved when comparisons are made across species or tasks. Here, humans completed the task, on a computer, for extra course credit, whereas the mice were hungry and ran for access to a sweetened cereal. Despite these differences, our results were strikingly similar across species, especially in terms of the robust gender effect. This supports the claim that a similar underlying mechanism is being tapped in the two species. It is interesting to note that, in over a century of research on maze learning in many species, we could find only one paper (Heron \& Hunter, 1922) that makes direct comparisons between human and rodent behavior, despite the assumption, made by many researchers, that findings with rodents generalize to humans. The present results lend support to this claim and, more importantly, provide a tool by which to reconfirm this assumption in various special populations.

The gender differences provide a validation of the virtual reality method, since it replicates a large body of research showing such differences in a wide range of tasks (Galea \& Kimura, 1993; Gron et al., 2000; Harris, 1978; Voyer, Voyer, \& Bryden, 1995). These differences are exemplified in Figure 2, where paths for each subject are superimposed one on top of another for Mazes 5 and $11 .^{2}$ Females chose longer paths and made more choices down blind alleys. As well, there were more pauses of longer duration for females than for males. The pauses observed with the humans are reminiscent of the phenomenon first noted by Tolman (1948) and labeled vicarious trial and error, which was observed at choice points in the behavior of maze-solving rats. It is as if, on the first trial of every maze, the women perceive more choice points than do the men and stop to examine their environment. Meanwhile, men make fewer pauses and choose a more direct route to the goal box. This may be related to the observation that men tend to use Euclidean representations, whereas women rely more on landmarks and verbally mediated route learning (Dabbs, Chang, Strong, \& Milun, 1998; Harris, 1978; Lawton, 1994). In the present context, in the first trial of every maze, men would benefit more from prior knowledge of where the goal box is relative to their present location, whereas, with a less rich representation, women would rely more on exploratory behaviors.

Recent data using functional magnetic resonance imaging, collected while subjects traversed a virtual maze (Gron et al., 2000), are consistent with this proposal. Specifically, these results showed that different brain areas were activated in men and women. For men, the unique activation occurred in the left hippocampus, whereas for women there was more activity in the right parietal and prefrontal cortexes. The hippocampus is known to be involved in the representation of external space (Nadel, 1980), whereas the fronto-parietal network has been implicated in spatial working memory (Jonides et al., 1993).

Finally, the present technology makes the use of virtual environments readily adapted for use with patient populations (e.g., Brooks et al., 1999; Skelton, Bukach, Laurance, Thomas, \& Jacobs, 2000). The interface is relatively simple, and the environment is rich enough to provide a sense of depth without being immersive, which can produce nausea and disorientation (cf. Nichols, 1999). The program we have developed ${ }^{3}$ can be configured to present spatial layouts of varying complexity based on simple stimulus file parameters.

The goal of the present work was to present a single tool that can be used as a cognitive test battery for both humans and mice. Previous attempts to develop rodent test batteries that include cognitive phenotyping have met with mixed success (Crawley et al., 1997; Crawley \& Paylor, 1997; Crusio, 1999). Here, we reverse engineered a standard and popular rodent test of intelligence and applied it to humans, thus providing an arena for direct comparisons. This, in some sense, brings full circle the literature on maze learning, which had been proposed as a nonlingustic test of general human intelligence (Porteus, 1950).

\section{REFERENCES}

Astur, R. S., Ortiz, M. L., \& Sutherland, R. J. (1998). A characterization of performance by men and women in a virtual Morris water task: A large and reliable sex difference. Behavioural Brain Research, 93, 185-190.

Brooks, B. M., McNeil, J. E., Rose, F. D., Greenwood, R. J., Attree, E. A., \& Leadbetter, A. G. (1999). Route learning in a case of amnesia: A preliminary investigation into the efficacy of training in a virtual environment. Neuropsychological Rehabilitation, 9, 63-76.

Clements, R. L., \& Brown, R. E. (1999). 100 years of mazes in psychology \& neuroscience. Society for Neuroscience Abstracts, 25, 261. 
Crawley, J. N., Belknap, J. K., Collins, A., Crabbe, J. C., Frankel, W., Henderson, N., Hit zemann, R. J., Maxson, S. C., Miner, L. L., Silva, A. J., Wehner, J. M., Wynshaw-Boris, A., \& Paylor, R. (1997). Behavioral phenotypes of inbred mouse strains: Implications and recommendations for molecular studies. Psychopharmacology, 132, 107-124.

Crawley, J. N., \& Paylor, R. (1997). A proposed test battery and constellations of specific behavioral paradigms to investigate the behavioral phenotypes of transgenic and knockout mice. Hormones \& Behavior, 31, 197-211.

Crusio, W. E. (1999). Using spontaneous and induced mutations to dissect brain and behavior genetically. Trends in Neurosciences, 22, 100-102.

Dabbs, J. M., Chang, E. L., Strong, R. A., \& Milun, R. (1998). Spatial ability, navigation strategy, and geographic knowledge among men and women. Evolution of Human Behavior, 19, 89-98.

DARKEN, R. P., \& SiberT, J. L. (1996). Navigating large virtual spaces. International Journal of Human-Computer Interaction, 8, 49-71.

Davenport, J. W., Hagquist, W. W., \& Rankin, G. R. (1970). The symmetrical maze: An automated closed-field test series for rats. Behavior Research Methods \& Instrumentation, 2, 112-119.

Emilien, G., Maloteaux, J.-M., Bey reuther, K., \& Masters, C. L. (2000). Alzheimer disease: Mouse models pave the way for therapeutic opportunities. Archives of Neurology, 57, 176-181.

Galea, L. A., \& Kimura, D. (1993). Sex differences in route-learning. Personality \& Individual Differences, 14, 53-65.

Gillner, S., \& MAllot, H. A. (1998). Navigation and acquisition of spatial knowledge in a virtual maze. Journal of Cognitive Neuroscience, 10, 445-463.

Gron, G., Wunderlich, A. P., Spitzer, M., Tomczak, R., \& Riepe, M. W. (2000). Brain activation during human navigation: Genderdifferent neural networks as substrate of performance. Nature Neuroscience, 3, 404-408.

Harris, L. J. (1978). Sex differences in spatial ability: Possible environmental, genetic, and neurological factors. In M. Kinsbourne(Ed.), Asymmetrical function of the brain (pp. 405-522). Cambridge: Cambridge University Press.

Heвb, D. O., \& Williams, K. A. (1946). A method of rating animal intelligence. Journal of General Psychology, 34, 59-65.

Heron, W. T., \& Hunter, W. S. (1922). Studies of the reliability of the problem box and the maze with human and animal subjects (Vol. 1). Baltimore: Williams \& Wilkins.

Jacobs, W. J., Laurance, H. E., \& Thomas, K. G. F. (1997). Place learning in virtual space: I. Acquisition, overshadowing, and transfer. Learning \& Motivation, 28, 521-541.

Jacobs, W. J., Thomas, K. G. F., Laurance, H. E., \& Nadel, L. (1998). Place learning in virtual space: Topographical relations as one dimension of stimulus control. Learning \& Motivation, 29, 288-308.

Jonides, J., Smith, E. E., Koeppe, R. A., Awh, E., Minoshima, S., \& Mintun, M. A. (1993). Spatial working memory in humans as revealed by PET. Nature, 363, 623-625.

LAwTON, C. A. (1994). Gender differences in way-finding strategies: Relationship to spatial ability and spatial anxiety. Sex Roles, 30, 765-779.

Meunier, M., Saint-Marc, M., \& Destrade, C. (1986). The HebbWilliams test to assess recovery of learning after limbic lesions in mice. Physiology \& Behavior, 37, 909-913.

Moffat, S. D., Hampson, E., \& Hatzipantelis, M. (1998). Navigation in a "virtual" maze: Sex differences and correlation with psychometric measures of spatial ability in humans. Evolution of Human Behavior, 19, 73-87.
NAdel, L. (1980). Cognitive and neural maps. In P. W. Jusczyk \& R. M. Klein (Eds.), The nature of thought: Essays in honor of D. O. Hebb (pp. 207-229). Hillsdale, NJ: Erlbaum.

Nichols, S. (1999). Physical ergonomics of virtual environment use. Applied Ergonomics, 30, 79-90.

Olton, D. S. (1979). Mazes, maps, and memory. American Psychologist, 34, 583-596.

Olton, D. S., \& PAPAs, B. C. (1979). Spatial memory and hippocampal function. Neuropsychologia, 17, 669-682.

Pollard, J. S., \& Sampson, H. (1961). Scoring performance in the closed field test. Australian Journal of Psychology, 13, 92-98.

Porteus, S. D. (1950). The Porteus maze test and intelligence. Palo Alto, CA: Pacific Books.

Rabinovitch, M. S., \& Rosvold, H. E. (1951). A closed field intelligence test for rats. Canadian Journal of Psychology, 5, 122-128.

Sandstrom, N. J., Kaufman, J., \& Huettel, S. A. (1998). Males and females use different distal cues in a virtual environment navigation task. Cognitive Brain Research, 6, 351-360.

Skelton, R. W., Bukach, C. M., Laurance, H. E., Thomas, K. G. F., JACOBS, W. J. (2000). Humans with traumatic brain injuries show place-learning deficits in computer-generated virtual space. Journal of Clinical \& Experimental Neuropsychology, 22, 157-175.

Tolman, E. C. (1948). Cognitive maps in rats and men. Psychological Review, 55, 189-208.

VAN Leuven, F. (2000). Single and multiple transgenic mice as models for Alzheimer's disease. Progress in Neurobiology, 61, 305-312.

Voyer, D., Voyer, S., \& Bryden, M. P. (1995). Magnitude of sex differences in spatial abilities: A meta-analysis and consideration of critical variables. Psychological Bulletin, 117, 250-270.

\section{NOTES}

1. Three sources of evidence can be used to argue against the possibility that the gender differences in virtual environments may stem from differential experience with such environments and with computer use in general. First, compare Trial 5 from Maze 11 with the earlier trials from this maze (Figure 2B). If the gender difference was caused by inefficiency in computer interfacing, Trial 5 would show a similar pattern to the previous four trials, which is not the case. Second, we administered questionnaires to assess (1) general computer experience and (2) 3-D gaming experience. We found clear gender differences in both measures, with men having more experience with both. However, a linear stepwise regression indicated that these two factors did not account for a significant proportion of the variance for overall performance, whereas gender $\operatorname{did}(R=.49, p<.05)$. Finally, the magnitude and nature of the gender differences in both acquisition and testing were highly similar across species, and the mice we tested had no computer experience!

2. A similar figure for 6 individuals can be viewed on the World-Wide Web (www.mcmaster.ca/dshore/hebb), along with a movie showing their way-finding behavior for Maze 5. These individuals are the best, median, and worst men and women.

3. Programming of the maze was done by W.J.M., who would be happy to make it available for academic research under the GNU public license.

(Manuscript received November 22, 2000; revision accepted for publication January 19, 2001.) 